- Nature. The EPA process for reviewing air-quality standards is focused on "eliciting, synthesizing, and documenting the opinions and judgments" of agency scientists, which are often based on "ambiguous statistical associations that depended on unverified models and assumptions", he said. Cox's own research has questioned the link between reducing fineparticle pollution and saving lives.

\section{BURDEN OF PROOF}

But there is mounting evidence, compiled by scientists from around the world, linking pollution to higher death rates. In a 2017 study of almost 61 million people, for instance, researchers at Harvard University in Cambridge, Massachusetts, used satellite data and computer models to map out daily pollution levels on a 1 kilometre $\times 1$ kilometre grid across the United States for 12 years (Q. Di et al. N. Engl. J. Med. 376, 2513-2522; 2017). After controlling for factors such as income, the scientists found that death rates increased in areas with more fine-particulate pollution and higher levels of ozone, a major component of smog even if those regions met air-quality standards.

If anything, those results suggest that the national standard should be stricter than it is now, says Francesca Dominici, a biostatistician at Harvard University and a co-author of the 2017 study, as well as the Science editorial.

Cory Zigler, a biostatistician at the University of Texas at Austin, says that Cox has effectively declared his own statistical methods king, writing off a variety of studies demonstrating the link between air pollution and public health.

Cox says he is aware of such criticisms and that he is only following the science where it leads, regardless of political consequences. "My sole motivation and commitment is to uphold and apply good science," he told Nature.

However, researchers including Christopher Frey, an environmental engineer at North Carolina State University in Raleigh, have pointed out that the current CASAC lacks the scientific expertise to properly evaluate the EPA's work on air-quality standards. Frey is a former CASAC chair and was on the scientific review panel that was dissolved last year.

Cox and other CASAC members have publicly acknowledged this criticism and say that they need access to additional expertise. During the 28 March meeting, CASAC revised its draft letter requesting that the EPA reinstate the previous review panel or create a new one.

What happens next is unclear. Normally, the EPA would revise its evaluation of the scientific research on the pollutant in question after input from CASAC and the scientific advisory panel. Then the agency would assess any health risks and exposure trends. If the EPA found that an update to the standard was justified, it would formally propose a change. But many scientists and environmentalists expect that the EPA will try to consolidate these steps to finalize a new standard next year.

\section{LIGO restarts with quantum boost}

\section{Detailed data on gravitational waves are set to pour in from the US detector and its European cousin, Virgo.}

\section{BY DAVIDE CASTELVECCHI}

$\mathrm{T}$ he hunt for gravitational waves is on again - this time assisted by the quirks of quantum mechanics.

Three massive detectors - two in the United States called LIGO and one in Italy known as Virgo - resumed collecting data on 1 April, after a 19-month shutdown for upgrades. Thanks in part to a quantum phenomenon known as light squeezing, the machines promise not only to spot more gravitational waves - ripples in space-time that can reveal a wealth of information about the cosmos - but also to make more detailed detections than before. Researchers hope to observe as-yet undetected events, such as a supernova or the merging of a black hole with a neutron star.

The run, which will last until March, also marks a major change in how gravitationalwave astronomy is done. For the first time, LIGO and Virgo will send out public, realtime alerts on wave detections to tip off other observatories - and anyone with a telescope - on how to find the events, so that they can be studied in different parts of the light spectrum. "Astronomers are really hungry," says David Reitze, a physicist at the California Institute of Technology in Pasadena and director of LIGO (the Laser Interferometer
Gravitational-wave Observatory), which made the first detection of gravitational waves in 2015.

In their previous two observing runs, LIGO's twin detectors spotted 11 gravitational-wave signals, each emanating from an epic cosmic collision - 10 of them from mergers between two black holes. The Virgo detector joined the network in 2017 and made contributions to several detections - in particular, to the first sighting, in 2017, of waves created by two merging neutron stars.

The upgraded network should be able to detect an average of one event per week, up from one a month, says Reitze. Most waves will probably be from black-hole mergers, but physicists are eager to see another neutron-star collision.

The increased sensitivity will enable the detectors to better discern signals from background noise, offering physicists more detail on the waves. This could allow for precise tests of the general theory of relativity, which predicted the existence of gravitational waves.

Future detections should reveal secrets about black holes that are in the process of merging, such as how fast they spin and in which direction, says Ilya Mandel, a theoretical astrophysicist at Monash University in Melbourne, Australia. "Maybe we can start teasing out some information about whether

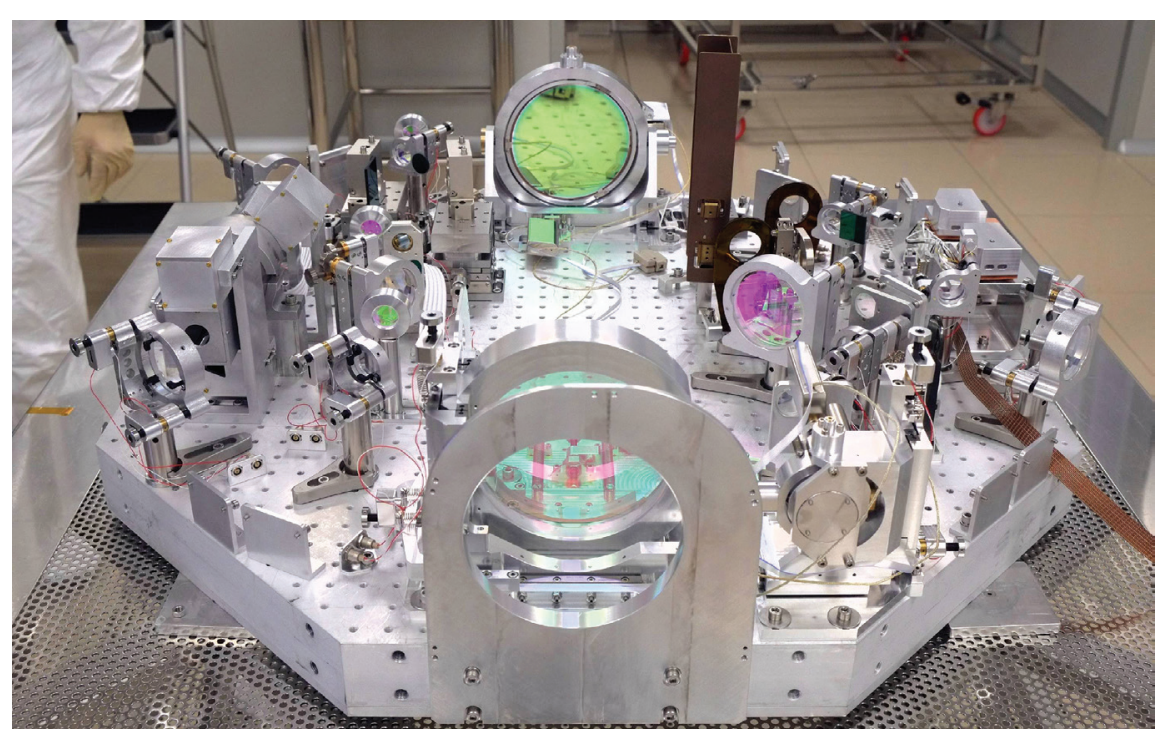

A part of Virgo, a gravitational-wave detector in Italy that now has nearly twice its previous sensitivity. 
they preferentially align," he says.

The upgrades have boosted the sensitivity of LIGO's machine in Livingston, Louisiana - already the most sensitive detector - by $40 \%$. The other LIGO interferometer, in Hanford, Washington, and Virgo near Pisa were hampered by technical snags in 2017 but have now partially caught up. Since 2017, Virgo has roughly doubled the distance within which it can detect events, says Alessio Rocchi, its commissioning coordinator and a physicist at the National Institute for Nuclear Physics in Rome.

\section{LASER UPDATES}

The sensitivity boosts result largely from two changes in the lasers at the heart of the observatories. Each LIGO detector is an L-shaped vacuum system that stretches over two 4-kilometre-long arms; the Virgo machine is similar, but has 3-kilometre arms. Inside, laser beams bounce between mirrors at both ends. When gravitational ripples pass through Earth, they cause the beams to change slightly in length.

To make signals stand out better from noise, LIGO and Virgo physicists have ramped up the lasers' power and have for the first time deployed a technique called squeezed light, which is based on a quirk of quantum mechanics.

Empty space bubbles with elementary particles that continually come into existence only to disappear moments later. These random fluctuations cause photons in the laser beams of gravitational-wave detectors to hit the mirrors at unpredictable times. At LIGO and Virgo, this has been the main hurdle to detecting gravitational waves of a high frequency, or pitch. But physicists can use squeezed light to shift some fluctuations towards lower frequencies to improve the detection of high-frequency waves.

The technique could particularly improve the detection of waves created by merging binary neutron stars or small black holes. That's because, as the objects spiral into each other, the lighter ones circle each other up to 500 times per second right before they collide, and their waves become so high-pitched that they fall out of the interferometers' range. Higher sensitivity could enable the detectors to track the objects all the way to their fiery end.

Astronomers around the world are also preparing to follow up on wave detections and to examine the same events using conventional facilities - including radio and X-ray observatories - thanks to the alert system, which will also be available through a smartphone app. In previous runs, teams that wanted to do such follow-ups had to sign memoranda of understanding with the LIGO-Virgo collaboration to receive confidential alerts. Now, there is no restriction, Reitze says. “That's a big change." -

\section{Iranian spying trial prompts global outcry}

\section{Reports allege that wildlife experts charged with espionage are being denied legal rights.}

\section{A}

BY MICHELE CATANZARO controversy surrounding eight environmental researchers charged with spying in Iran is escalating. Leading conservation groups, human-rights organizations and European politicians are urging Iranian authorities to give the researchers a fair trial, after reports emerged that the defendants' legal rights have been breached. Meanwhile, secretive trials of the scientists - who say they are innocent - have begun in Iran's revolutionary court system, which usually deals with economic, corruption and security crimes, according to sources close to the defendants. Four of the researchers face extra charges of "sowing corruption on Earth", which can carry the death penalty.

The sources, who asked to remain anonymous for fear of retaliation, say that hearings have been held for two defendants, after an initial session in which the indictment was read to the whole group. The sources also say that two researchers are having health problems because they haven't received proper care in prison.

The case comes amid increasing scrutiny by the Iranian government of environmentalresearch activities. Critics say the government fears that other nations might be using environmental monitoring as a cover for spying, and according to the Center for Human Rights

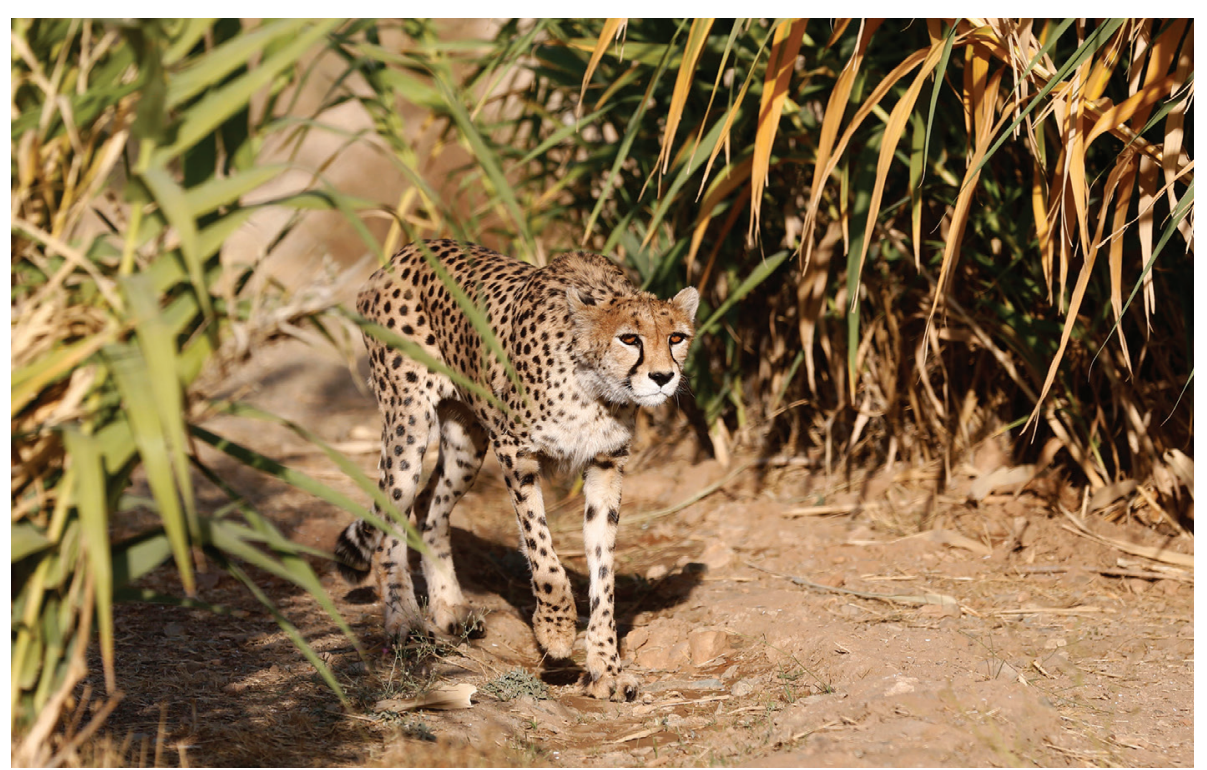

An Asiatic cheetah. Researchers studying these endangered cats in Iran have been arrested and jailed.

in Iran, a non-governmental organization headquartered in New York City, tens of people involved in conservation have been arrested in Iran in the past year.

"Environment was a safe space in Iran, because it's apolitical," says Kaveh Madani, an environmental-policy specialist at Imperial College London. But increasing interest in the nation's worsening environmental issues from both the Iranian public and Western conservation organizations has changed that, he says.

Many conservation researchers now fear working in Iran, says Madani, who is Iranian and was invited back to Iran from London in 2017 to serve as head of the government's environment department. But he resigned 